\title{
Electric Vehicle Charging in Smart Grid: Optimality and Valley-filling Algorithms
}

\author{
Niangjun Chen*, Student Member, IEEE, Chee Wei Tan, Senior Member, IEEE, and \\ Tony Q. S. Quek, Senior Member, IEEE.
}

\begin{abstract}
Electric vehicles (EVs) offer an attractive long-term solution to reduce the dependence on fossil fuel and greenhouse gas emission. At the same time, charging a large fleet of EVs distributed across the residential area poses a challenge for the distribution network. In this paper, we formulate this problem by building on the optimal power flow (OPF) framework to model the network constraints that arises from charging EVs at different locations. To overcome the computational challenge when the control horizon is long, we study a nested optimization approach to decompose the joint OPF and EV charging problem. We characterize the optimal EV charging schedule to be a valleyfilling profile, which allows us to develop an efficient offline algorithm with significantly lower computational complexity compared to centralized interior point solvers. Furthermore, we propose a decentralized online algorithm that dynamically tracks the valley-filling profile. Our algorithms are evaluated on the IEEE 14 bus system with real residential load profiles, and the simulations show that our online algorithm performs almost optimally under different settings.
\end{abstract}

Index Terms-Optimal power flow, electric vehicle charging, valley-filling, online algorithm, convex optimization.

\section{INTRODUCTION}

Electric vehicles (EVs) are getting more popular as a longterm vehicular technology in order to reduce the dependence on fossil fuel and the emission of greenhouse gases. However, as EV penetration increases, uncoordinated charging can lead to additional power losses and unacceptable voltage variation that may overload the power grid [1]. To tackle this problem, we can increase the power delivery capacity to deal with the new EV peak demands, but this will lead to significant infrastructure cost. On the other hand, we can adopt a "smart grid" solution, which allows EVs to communicate with the utility that in turn coordinates their charging activities. Besides preventing grid overload, a coordinated EV charging can improve frequency regulation [2], smooth out the generation intermittency from renewables, and increase the efficiency in electricity usage [3], [4].

In this paper, we consider two types of load that are connected to the power network:

- Price-inelastic load: The exact power requested must be provided. This corresponds to standard loads in a conventional grid such as lighting and heating.

The work in this paper was partially supported by grants from the Research Grants Council of Hong Kong Project No. RGC CityU 122013 and SRG ISTD 2012037.

N. Chen is with the Department of Computing and Mathematical Sciences, California Institute of Technology, Pasadena, CA 91125 USA (email: ncchen@caltech.edu).

C. W. Tan is with the College of Science and Engineering, City University of Hong Kong, Kowloon, Hong Kong (e-mail: cheewtan Qcityu. edu.hk).

T. Q. S. Quek is with the Information Systems Technology \& Design Pillar, Singapore University of Technology \& Design and the Institute for Infocomm Research, Singapore (e-mail: tonyquek@ sutd.edu.sg) .
- Price-elastic load: The power delivered to this type of load can vary depending on the current cost and a deadline. An example is charging and recharging of EV batteries.

By considering these two types of loads, we study two key problems: First, how to minimize the total cost of power generation and EV charging? Second, how to deal with uncertainties in price-inelastic load in the online setting? To take into account the physical constraints in a power grid, we leverage the well-known optimal power flow (OPF) problem and consider its time-dependent extension, i.e., a multi-period $\mathrm{OPF}$.

The OPF problem optimizes the operation of a power grid subject to the voltage constraints, line constraints and power balance constraints. However, the OPF is nonconvex and NPhard in general [5]. Recently, it has been shown that most practical power configurations exhibit a surprisingly useful property that enables the OPF problem to be exactly solved by solving its convex relaxations under some conditions [5][8]. This convex relaxation methodology leads to the design of efficient polynomial time algorithms and can also be used to decompose the OPF into simpler subproblems, e.g., a multiperiod OPF with energy storage [9], [10].

\section{A. Summary of Contributions}

In this paper, we extend the OPF problem to a timedependent joint OPF-EV charging problem to account for the price-elastic load. We leverage the zero duality gap result in [5] to develop both offline and online algorithms that solve this joint OPF-EV charging optimization problem. To this end, we propose a nested optimization approach that decomposes the joint OPF-EV charging problem into separable subproblems, and then solve the decomposed problem using a nonsmooth separable programming technique. The main contribution of the paper are as follows:

1) We characterize the optimal solution to be valley-filling. This valley-filling characterization holds for all the network configurations that guarantee the zero duality gap condition in the OPF problem.

2) We propose an offline algorithm that can solve the joint OPF-EV charging problem with a computational complexity significantly lower than the centralized interior point solvers.

3) To account for the causality constraint from the priceinelastic load, we propose an online algorithm that dynamically tracks this valley-filling characteristic. We show that our online algorithm can be easily implemented in a decentralized manner. Simulation results show that the performance of our online algorithm is near optimal. 
There is a growing literature on various aspects of the EV charging problem. For example, the problem of charging EVs at centrally-managed large scale charging facilities is considered in [11], [12] by assuming arbitrary EV load arrival. The authors in [11], [12] design algorithm to achieve the best competitive ratio against the optimal offline problem; Examples of distributed EV charging protocols for residential areas is considered in [13], [14], where the goal of optimization is to minimize the operation cost. The authors in [15] propose distributed charging algorithm to maximize user utility and achieve proportional fairness. The integration of renewable energy in EV charging is considered in [11], [16]. The authors of [17].The authors in [9] formulate an offline EV charging problem using the full power flow constraints and propose to solve this centralized problem using semidefinite programming (SDP).

This work falls into the category of online distributed control for the EV charging problem from the perspective of the utility. The utility controls the elastic load via demand response (DR) programs. Common approaches to demand response include direct load control (DLC) and time of use based pricing. An overview of demand response can be found in [18]. In particular, our work in paper focuses on the DLC approach, where EV owners are offered monetary rewards for allowing their EVs to respond to utility control signals. The cost we seek to minimize is the total cost of power grid operation and the charging costs of EVs. The power flow constraints that we use is similar to that of [9], but we develop simple distributed control algorithms that have significantly lower computational complexity than centralized SDP solvers. An aggregate valley-filling characterization for optimal EV charging profile is proposed in [13] when the underlying network model is ignored. Our optimality characterization applies to a more general setting by taking the underlying power flow optimization into account. A preliminary version of this work has appeared in [19], and this paper expands on [19] to provide new analytical results on the feasibility and performance of our online algorithm. Also, we conduct additional numerical experiments under different settings.

\section{B. Assumptions}

We now make the following assumptions used in this paper.

- The EV charge points are equipped with low-latency two way communication with the utility;

- The charging points have capabilities to compute and adapt the charging rate at real time;

- The EV charging cost is time-invariant, i.e., the price per unit energy is fixed at the beginning (different charging stations can set different prices), but this cost does not vary over the charging period;

- An EV battery can be charged at any rate between the maximum and minimum charging rate ${ }^{1}$;

- The utility has an accurate estimate of the average EV charging load over the control horizon, and the charging points have good estimate of the maximum and minimum charging rates available in the future.

We assume that the online information arises from the uncertainties in the price-inelastic loads, but the EV charge points have good estimation of the EV loads. This model is suitable

\footnotetext{
${ }^{1}$ This is a widely assumed model in the literature, see, e.g. [13], [15], [20].
}

TABLE I: Notations

\begin{tabular}{|cl|}
\hline $\mathcal{N}$ & set of buses in the power grid network \\
$\mathcal{G}$ & set of generator buses in the grid \\
$\mathcal{L}$ & set of transmission lines in the network \\
$\mathbf{Y}$ & admittance matrix \\
$f_{k}(\cdot)$ & convex cost function for bus $k$ \\
$\mathbf{p}[t]+\mathbf{q}[t] \mathbf{j}$ & vector of power generated at time $t$ \\
$\tilde{\mathbf{p}}[t]+\tilde{\mathbf{q}}[t] \mathbf{j}$ & vector of price-inelastic demand at time $t$ \\
$\hat{\mathbf{p}}[t]$ & EV charging rate at time $t$ \\
$\boldsymbol{\alpha}$ & real vector of EV charging cost \\
$\mathbf{v}[t]$ & complex voltage vector at time $t$ \\
$\mathbf{W}[t]$ & $\mathbf{v}[t] \mathbf{v}[t]^{*}$ \\
$\mathbf{\mathbf { r }}[t], \underline{\mathbf{r}}[t]$ & EV charging rate limits at time $t$ \\
$\mathbf{c}$ & vector of total EV energy demand \\
$e_{k}$ & $k^{t h}$ standard basis of $\mathbf{R}^{|\mathcal{N}|}$ \\
\hline
\end{tabular}

for utilities controlling the EV charging points at residential area or parking facility near office building where the arrivals and departures of EVs are relatively static in equilibrium.

In this paper, we only consider minimizing the cost related to the active power. In a more general setting, utility may also have cost associated to reactive power and use the DLC approach to control devices that can absorb or inject active and reactive power [21]. However, the characteristics and constraints of these devices may be considerably different from the EVs. For simplicity of presentation and analysis, we thus focus only on the cost related to the active power.

The rest of the paper is organized as follows. The system model and problem formulation is introduced in Section II. The main analytical results and valley-filling algorithms are given in section III and IV respectively. Numerical evaluations are given in Section V. Finally, the conclusions are given in Section VI. Table I summarizes the notation we use throughout the paper. We use lower case letters for scalars, blocked lower case letters for vectors, blocked upper case letter for matrices, and use $(\mathbf{x}[t])_{j}$ to represent the value of the $j^{\text {th }}$ component of a vector $\mathbf{x}[t]$ at iteration $t$.

\section{System Model AND Problem Formulation}

We consider a discrete-time model where each time interval represents the timescale at which the power grid adjusts its power generation. The goal is to optimize the operation of the power grid over a time-interval of interest $t \in\{0,1, \ldots, T\}$. In practice, $T$ could be the duration of a day and a timeslot $t$ could be in the order of minutes. In addition, we assume that price-inelastic loads are fixed over each time interval $[t, t+1]$.

\section{A. EV Load Model}

Suppose that each bus $k \in \mathcal{N}$ can connect to a priceinelastic load and a price-elastic EV battery. Furthermore, we assume that each EV battery can absorb or inject only active power at an adjustable rate via a smart outlet. In the following, we assume that each bus is connected to only one EV battery. However, our results can be generalized to the case when multiple EV batteries are co-located at the same bus. We consider the following two types of constraints associated with EV loads:

1) Charging rate Each smart outlet has a charging rate limit at each time $t$ [20], [22], hence for $k \in \mathcal{N}$ and $t \in$ $\{1,2, \ldots, T-1\}$,

$$
(\underline{\mathbf{r}}[t])_{k} \leq(\hat{\mathbf{p}}[t])_{k} \leq(\overline{\mathbf{r}}[t])_{k} .
$$


2) Deadline Each EV can have different arrival time and deadline. We model these constraints by setting $(\underline{\mathbf{r}}[t])_{k}=(\overline{\mathbf{r}}[t])_{k}=0$ if $\mathrm{EV}$ has not arrived at bus $k$ at time $t$, or the charging deadline of $\mathrm{EV}$ at bus $k$ has passed.

Let $B_{k}, s_{k}(0)$, and $\eta_{k}$ denote the battery capacity, initial state of charge (SOC), and charging efficiency, respectively. By the deadline $T$, the EV should be fully charged, hence, $\eta_{k} \sum_{t=1}^{T-1}(\hat{\mathbf{p}}[t])_{k} \Delta t=B_{k}\left(1-s_{k}(0)\right)$. Let $C_{k}:=B_{k}\left(1-s_{k}(0)\right) /\left(\eta_{k} \Delta t\right)$, then the EV charging constraint is the following, for all $k \in \mathcal{N}$

$$
\sum_{t=1}^{T-1}(\hat{\mathbf{p}}[t])_{k}=\mathbf{c}_{k} .
$$

\section{B. Network Structure}

Consider a power network with a set of buses $\mathcal{N}:=$ $\{1, \ldots, n\}$, a set of generator buses $\mathcal{G} \subseteq \mathcal{N}$, and a set of flow lines $\mathcal{L} \subseteq \mathcal{N} \times \mathcal{N}$. Let $z_{l m}$ and $y_{l m}$ be the complex impedance and admittance between bus $l$ and $m$, respectively, and we have $y_{l m}=\frac{1}{z_{l m}}$. Denote $\mathbf{Y}=\left(Y_{l m}, 1 \leq\right.$ $l, m \leq n)$ as the admittance matrix and let column vectors $\mathbf{v}=\left(V_{1}, V_{2}, \ldots, V_{|\mathcal{N}|}\right)^{\top} \in \mathbb{C}^{|\mathcal{N}|}$ and $\mathbf{i}=\left(I_{1}, I_{2}, \ldots, I_{|\mathcal{N}|}\right)^{\top} \in$ $\mathbb{C}^{|\mathcal{N}|}$ be the voltage and current vectors, respectively. By Ohm's Law and Kirchoff's Current Law, we have $\mathbf{i}=\mathbf{Y} \mathbf{v}$.

Define $e_{k}$ to be the $k^{t h}$ standard basis in $R^{\mathcal{N}}$, then the power injection at bus $k$ at time $t$ can be written as

$$
(\mathbf{v}[t])_{k}(\mathbf{i}[t])_{k}=\operatorname{Tr}\left\{\mathbf{v}[t] \mathbf{v}[t]^{*} Y^{*} e_{k} e_{k}^{*}\right\}=\operatorname{Tr}\left\{\mathbf{W}[t] Y^{*} e_{k} e_{k}^{*}\right\} .
$$

\section{Joint OPF-EV Charging Optimization Problem}

The OPF problem finds the stable operating point that minimizes an appropriate cost function, for example generation cost or power loss, subject to certain physical constraints on the power and voltage variables [23]. Using the EV load model, we augment the OPF problem to the following timedependent joint OPF-EV charging optimization problem:

$$
\begin{aligned}
\min _{\{\mathbf{W}[t], \hat{\mathbf{p}}[t]\}} & \sum_{t=1}^{T-1} \sum_{k \in \mathcal{G}} f_{k}\left((\mathbf{p}[t])_{k}\right)+\sum_{t=1}^{T-1} \sum_{k \in \mathcal{N}}(\boldsymbol{\alpha})_{k}(\hat{\mathbf{p}}[t])_{k} \\
\text { s.t. } \quad & P_{k}^{\min } \leq(\mathbf{p}[t])_{k} \leq P_{k}^{\max }, \\
& Q_{k}^{\min } \leq(\mathbf{q}[t])_{k} \leq Q_{k}^{\max }, \\
& \left(V_{k}^{\min }\right)^{2} \leq \mathbf{W}[t]_{k k} \leq\left(V_{k}^{\max }\right)^{2}, \\
& \left(\mathbf{W}[t]_{l l}-\mathbf{W}[t]_{l m}\right) y_{l m}^{*} \leq S_{l m}^{\max }, \\
& \operatorname{Tr}\left\{\mathbf{W}[t] \mathbf{Y}^{*} e_{k} e_{k}^{*}\right\}=\left((\mathbf{p}[t])_{k}-(\hat{\mathbf{p}}[t])_{k}-(\tilde{\mathbf{p}}[t])_{k}\right) \\
& +\left((\mathbf{q}[t])_{k}-(\tilde{\mathbf{q}}[t])_{k}\right) \mathbf{j}, \\
& \mathbf{W}[t] \succeq 0, \\
& \operatorname{rank}(\mathbf{W}[t])=1, \\
& T-1 \\
& \sum_{t=1} \hat{\mathbf{p}}[t]=\mathbf{c}, \\
& \underline{\mathbf{r}}[t] \leq \hat{\mathbf{p}}[t] \leq \overline{\mathbf{r}}[t],
\end{aligned}
$$

for every $k \in \mathcal{N}$ and $(l, m) \in \mathcal{L}$, where (3b), (3c) are the power generation constraints, $(3 \mathrm{~d})$ is the voltage magnitude constraint, (3e) is the line flow constraint, and (3f) is the power balance constraint.
The cost term $f_{k}\left((\mathbf{p}[t])_{k}\right)$ can be considered as positive convex cost or negative concave utility function as specified in [7]. The objective function can be interpreted as social welfare as it considers both the power generation cost and the EV charging cost. The utility may offer minimizing the $\mathrm{EV}$ charging cost as an added incentive for EV owners to join the demand response program, but it can also set the charging cost $\boldsymbol{\alpha}$ to be 0 if it emphasizes only the power generation cost.

Although we can solve (3) exactly by an SDP relaxation of the non-convex constraint (3h) [5] [6], this problem consists of $(T-1)$ instances of individual OPF problems with time varying constraints, and the decision variables are furthermore coupled by the EV charging constraint (3i). Hence, there will be $O((|\mathcal{N}|+|\mathcal{L}|) T)$ variables in the dual SDP problem of (3). For large $T$, the computational complexity of solving (3) as a whole by centralized interior point solvers may be prohibitive (the computational bottleneck of interior point solvers lies in the inversion of the Hessian matrix when the problem size scales up) [24], [25]. Hence, solving this problem using centralized SDP solver is not scalable for large $T$. In the following, we propose a way to efficiently solve (3) for large $T$ by decomposing it to smaller sub-problems.

\section{Optimal OfFline Algorithm For EV SCHEDUling PROBLEM}

In this section, we deal with the scalability problem of the joint OPF-EV charging problem in the offline setting. Assuming all the information about EV load and price-inelastic load in the control horizon is available, we exactly characterize the optimal EV charging profile and this characterization leads us to decompose the time-dependent problem over time. For clarity of presentation, the proofs of the theorems stated in the subsequent sections are presented in the Appendix.

\section{A. Decoupling Power Dispatching from EV Scheduling}

While the optimization variables in the Joint OPF-EV charging problem (3) are $\mathbf{W}[t]$ and $\hat{\mathbf{p}}[t]$, if the optimal charging decision $\hat{\mathbf{p}}^{*}[t]$ is known, then the remaining problem of finding $\mathbf{W}^{*}[t]$ is separable in $t$. To show this, we define the following function:

$$
\begin{array}{r}
F\left(\hat{\mathbf{p}}^{*}[t]+\tilde{\mathbf{p}}[t]\right):=\min _{\mathbf{W}[t]}\left(\sum_{k \in \mathcal{N}} f_{k}\left((\mathbf{p}[t])_{k}\right)\right) \\
\text { s.t. }(3 \mathrm{~b}),(3 \mathrm{c}), \ldots,(3 \mathrm{~h}),
\end{array}
$$

where $F\left(\hat{\mathbf{p}}^{*}[t]+\tilde{\mathbf{p}}[t]\right)$ returns the optimal value of the OPF problem for a total demand $\left(\hat{\mathbf{p}}^{*}[t]+\tilde{\mathbf{p}}[t]\right)$. Given $\hat{\mathbf{p}}^{*}[t]$, we can find $\mathbf{W}^{*}[t]$ by solving (4) alone. Since (4) is essentially an OPF problem with $O(|\mathcal{N}|+|\mathcal{L}|)$ variables in its convex dual, we can solve each $\mathbf{W}^{*}[t]$ efficiently. What remains is to find the optimal EV charging rate $\hat{\mathbf{p}}^{*}[t]$, which can be written as the optimal solution of the following EV scheduling problem:

$$
\begin{aligned}
\min _{\hat{\mathbf{p}}[t]} & \sum_{t=1}^{T-1} F(\hat{\mathbf{p}}[t]+\tilde{\mathbf{p}}[t])+\boldsymbol{\alpha}[t]^{\top} \hat{\mathbf{p}}[t] \\
\text { s.t. } & \underline{\mathbf{r}}[t] \leq \hat{\mathbf{p}}[t] \leq \overline{\mathbf{r}}[t] \quad \forall t \in[1, T-1], \\
& \sum_{t=1}^{T-1} \hat{\mathbf{p}}[t]=\mathbf{c} .
\end{aligned}
$$


As will be shown below, $F(\cdot)$ is convex, and this important property allows us to find the optimal solution $\hat{\mathbf{p}}^{*}[t]$ without having to solve $\mathbf{W}[t]$.

\section{B. Convexity of $F(\cdot)$}

The following result is a direct consequence of the zero duality gap property of the OPF problem, which reveals the convexity of the decoupled function in (4).

Theorem 1. If the zero duality gap holds, then $F: \mathbb{R}^{|\mathcal{N}|} \rightarrow \mathbb{R}$, is a convex function.

Remark 1. Although $F(\cdot)$ is convex, it can be nonsmooth. In general, $F(\cdot)$ is a pointwise supremum of convex functions.

In the following, we characterize the optimal solution of (5) by making use of the convexity of $F(\cdot)$ only, and does not make any assumption on its smoothness.

\section{Characterization of optimal offline solution}

By convexity of $F$, and suppose that the charging rate limits are inactive, we can apply Jensen's inequality and get the following result:

Lemma 1. If $\forall t, \underline{\mathbf{r}}[t]=-\infty, \overline{\mathbf{r}}[t]=\infty$, then the EV scheduling problem (5) has an optimal solution $\hat{\mathbf{p}}[t]=\mathbf{a}-\tilde{\mathbf{p}}[t]$, where $\mathbf{a}=\left(\sum_{t=1}^{T-1} \hat{\mathbf{p}}[t]+\tilde{\mathbf{p}}[t]\right) /(T-1)$ which is the average total load of the control horizon.

In this case, the optimal solution is a flat profile, i.e., $\forall t, \hat{\mathbf{p}}[t]+\tilde{\mathbf{p}}[t]$ is constant. Next, we consider the case where the charging rate constraints can be active. The optimal solution will then no longer be flat, but valley-filling as defined in the following:

Definition 1. A charging profile is valley-filling, if there exists a unique vector a, such that

$$
\hat{\mathbf{p}}[t]=[\mathbf{a}-\tilde{\mathbf{p}}[t]]_{\underline{\mathbf{r}}[t]}^{\overline{\mathbf{r}}[t]}, \forall t,
$$

where $[x]_{l}^{u}=\max (l, \min (x, u))$.

In the definition, a can be seen as a valley level that $\hat{\mathbf{p}}[t]+$ $\tilde{\mathbf{p}}[t]$ tries to reach unless $\hat{\mathbf{p}}[t]$ is constrained by $\overline{\mathbf{r}}[t]$ or $\underline{\mathbf{r}}[t]$. A similar definition of valley-filling for EV scheduling can be found in [13]. Interestingly, the valley-filling characterization is reminiscent of the water-filling notion for power allocation to maximize capacity in information theory [26].

The next result shows the optimality of a valley-filling profile. It can be proved by using a substitution argument, i.e., if there is an optimal charging profile that is not valley-filling, then by convexity of $F$, we can always construct a valleyfilling profile that has the same or lower objective value.

Theorem 2. For a general convex function $F(\cdot)$, a valleyfilling profile is optimal to the EV Scheduling problem (5).

The fact that we only need convexity of $F(\cdot)$ to prove Theorem 2 implies that, a valley-filling profile is a minimizer for any general convex objective, not just the objective of joint OPF-EV charging optimization as defined in (3a). For example, consider the following objective

$$
\sum_{t=1}^{T-1} F(\hat{\mathbf{p}}[t]+\tilde{\mathbf{p}}[t])=\sum_{t=1}^{T-1}\left(\sum_{k \in \mathcal{N}}\left((\hat{\mathbf{p}}[t])_{k}+(\tilde{\mathbf{p}}[t])_{k}\right)\right)^{2},
$$

which is the square of the $l_{2}$ norm of the aggregate load. As the aggregate load is constant, Theorem 2 implies that a valley-filling profile is also a load variance minimizing profile.

Next, we show the uniqueness of the valley level a. Note that a must satisfy the following for $j=1, \ldots,|\mathcal{N}|$ :

$$
\begin{gathered}
\min _{t}\left\{(\tilde{\mathbf{p}}[t])_{j}+(\underline{\mathbf{r}}[t])_{j}\right\} \leq \mathbf{a}_{j} \leq \max _{t}\left\{(\tilde{\mathbf{p}}[t])_{j}+(\overline{\mathbf{r}}[t])_{j}\right\} \\
\sum_{t=1}^{T-1} \hat{\mathbf{p}}[t]=\sum_{t=1}^{T-1}[\mathbf{a}-\tilde{\mathbf{p}}[t]]_{\underline{\mathbf{r}}[t]}^{\overline{\mathbf{r}}[t]}=\mathbf{c}
\end{gathered}
$$

Note that (7b) is a continuous and strictly increasing function for $\mathbf{a}_{j}$ for all $j$. Since (7b) is continuous and strictly increasing, we can find a unique a via the bisection method for the offline case. This is presented in the following algorithm with $\varepsilon$ as an error tolerance level. We determine a in a component-wise manner. Each iteration of the while loop will halve the search space for $\mathbf{a}_{j}$, therefore the computational complexity of the visection algorithm is low.

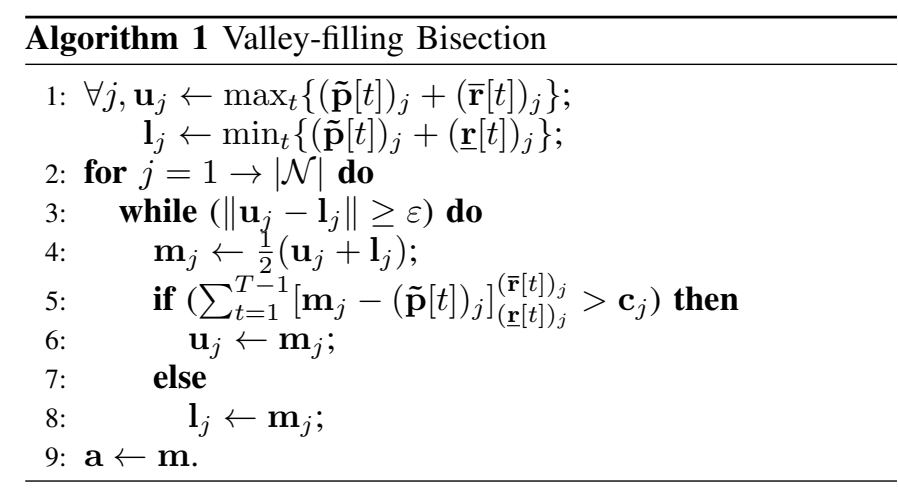

Once we have determined the value of $\mathbf{a}$, the scheduling of EV vehicle (solving $\hat{\mathbf{p}}[t]$ ) can now be done optimally in $O(1)$ time following the valley-filling characterization in Definition 1. Given the optimal $\hat{\mathbf{p}}[t]$, the remaining optimization variables $\mathbf{W}[t]$ become separable. The offline algorithm for the joint OPF-EV charging problem (3) is shown in Algorithm 2.

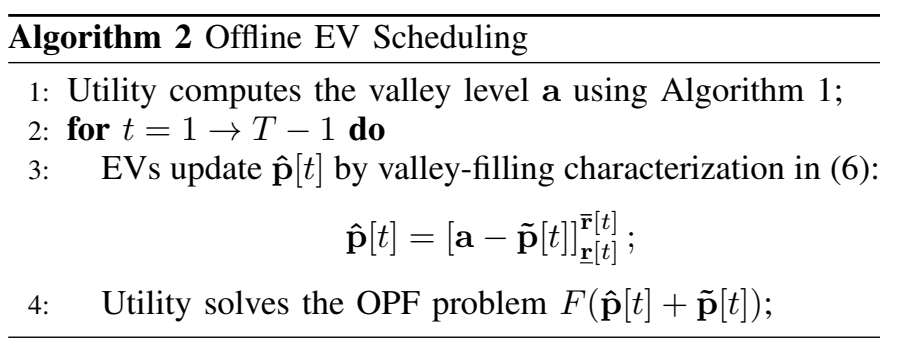

Algorithm 2 decomposes the joint OPF-EV charging problem (3) from an SDP with $O((|\mathcal{N}|+|\mathcal{L}|)(T-1))$ variables to $(T-1)$ SDPs each with $O(|\mathcal{N}|+|\mathcal{L}|)$ variables. The complexity of SDP interior point solvers grows superlinearly with respect to the number of variables [24], [25], thus this decomposition leads to a lower computational complexity. We show this reduction in computational complexity via simulation in section V-F.

\section{Online Algorithm for EV Scheduling Problem}

In this section, we build on the optimal characterization of EV charging schedule to design a practical online algorithm. 


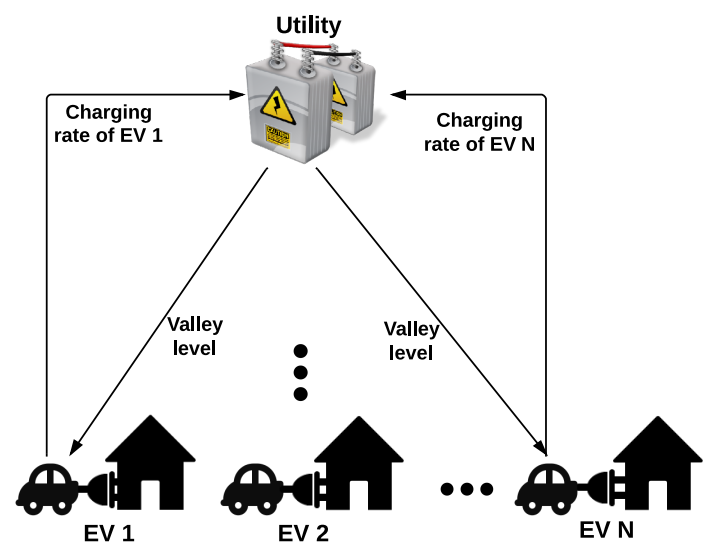

Fig. 1: An illustration of information flow between the utility and EV charge points at each time instance $t$, the utility broadcast the current valley level to all EVs, and each EV compute its charging rate and report back to the utility. Then utility will use the information gathered for computing the next valley level.

The description of our online EV scheduling algorithm is given in Algorithm 3. The information flow between EVs and the utility is illustrated in Fig. 1. Next we provide a narrative for the consideration of its implementation.

Under a causality constraint, we only know $\tilde{\mathbf{p}}[t]$ at time $t$. Therefore, in the online case, we cannot use the previous bisection algorithm to find a. Instead, we propose an algorithm that estimates the valley level, which is denoted by $\mathbf{a}^{\prime}[t]$ and adjusts it dynamically.

Let $\mathbf{p}_{\mathbf{E}}, \mathbf{p}_{\mathbf{I}}$ denote the average EV load and price-inelastic load respectively. From Lemma 1, the ideal valley level is $\left(\mathbf{p}_{\mathbf{E}}+\mathbf{p}_{\mathbf{I}}\right)$ if the charging rate constraints are not active. We will use the estimate of $\left(\mathbf{p}_{\mathbf{E}}+\mathbf{p}_{\mathbf{I}}\right)$ to set our initial valley level. We know the value of average EV load, $\mathbf{p}_{\mathbf{E}}=\mathbf{c} /(T-1)$, but the value of average inelastic load, $\mathbf{p}_{\mathbf{I}}$ has to be estimated, possibly by learning from historical record of the priceinelastic load.

Also, as we do not have complete information of the priceinelastic load fluctuations, following the valley level characterization of (6) alone may not guarantee that the resulting EV charging profile can meet the charging deadline. To deal with this, our online algorithm will detect the following events and deal with them separately:

- If from time $t$ onwards, even charging at the maximal rate will not fully charge the $\mathrm{EV}$ battery at bus $j$, i.e.,

$$
\sum_{k=1}^{t}(\hat{\mathbf{p}}[k])_{j}>\mathbf{c}_{j}-\sum_{l=t+1}^{T-1}(\underline{\mathbf{r}}[l])_{j},
$$

then it will raise an "undercharging" exception and increase the current charging rate.

- If from time $t$ onwards, even charging at the minimal rate will overcharge the EV battery at bus $j$, i.e.,

$$
\sum_{k=1}^{t}(\hat{\mathbf{p}}[k])_{j}>\mathbf{c}_{j}-\sum_{l=t+1}^{T-1}(\underline{\mathbf{r}}[l])_{j},
$$

then it will raise an "overcharging" exception and decrease the current charging rate.

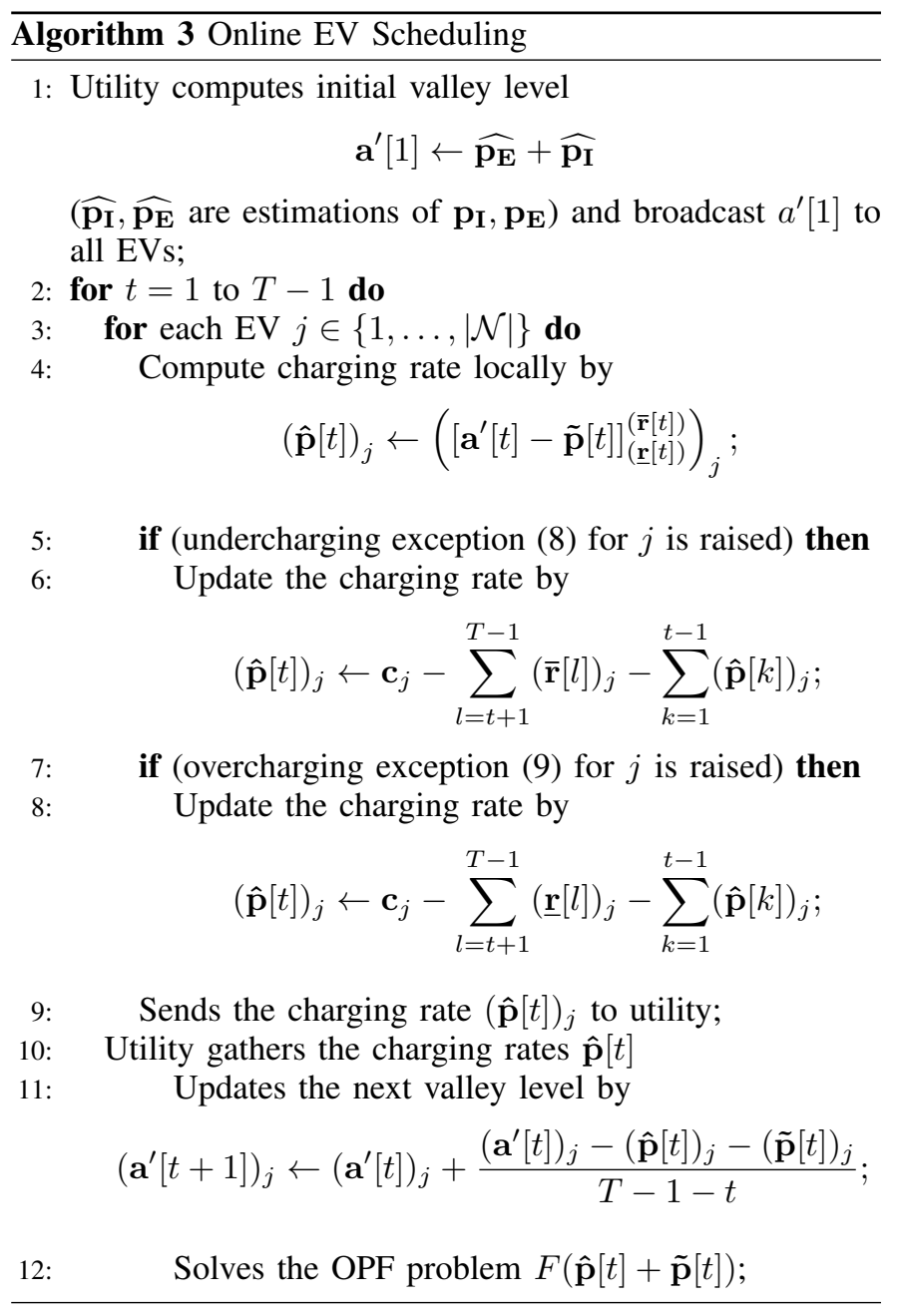

We can see from Algorithm 3 that, at each time $t$, each EV only needs to execute line 4 to 9 , which only takes $O(1)$, and the message communicated between utility and EVs are the valley-level a and the charging rate decision $\hat{\mathbf{p}}[t]$, hence the requirement on computation and communication capabilities for the charging points are relatively low.

Remark 2. Algorithm 3 only uses the prediction of average aggregate load to set the initial valley level, if the utility have accurate real time prediction price-inelastic load $\tilde{\mathbf{p}}[t]$, then we can use bisection algorithm like Algorithm 1 to get even better estimate of valley level $\mathbf{a}$.

The following result demonstrates that the charging schedule produced by Algorithm 3 is always feasible.

Theorem 3. If the charging rate constraints $(\overline{\mathbf{r}}[1], \ldots, \overline{\mathbf{r}}[T-$ $1])$ and $(\underline{\mathbf{r}}[1], \ldots, \underline{\mathbf{r}}[T-1])$ permit a feasible solution, then $(\hat{\mathbf{p}}[1], \ldots, \hat{\mathbf{p}}[T-1])$ obtained from Algorithm 3 is a feasible solution to $(5)$.

While line 5 to line 7 ensure feasibility, we may not want to trigger those exceptions, because once any of them holds true, subsequent charging rate will either stay at the maximum or the minimum (leaving no room for optimization). Line 11 helps to avoid the exceptions by updating the valley level estimation $\mathbf{a}^{\prime}[t]$ based on information up to time $t$. If the sum of the EV load and the price-inelastic load cannot meet the 


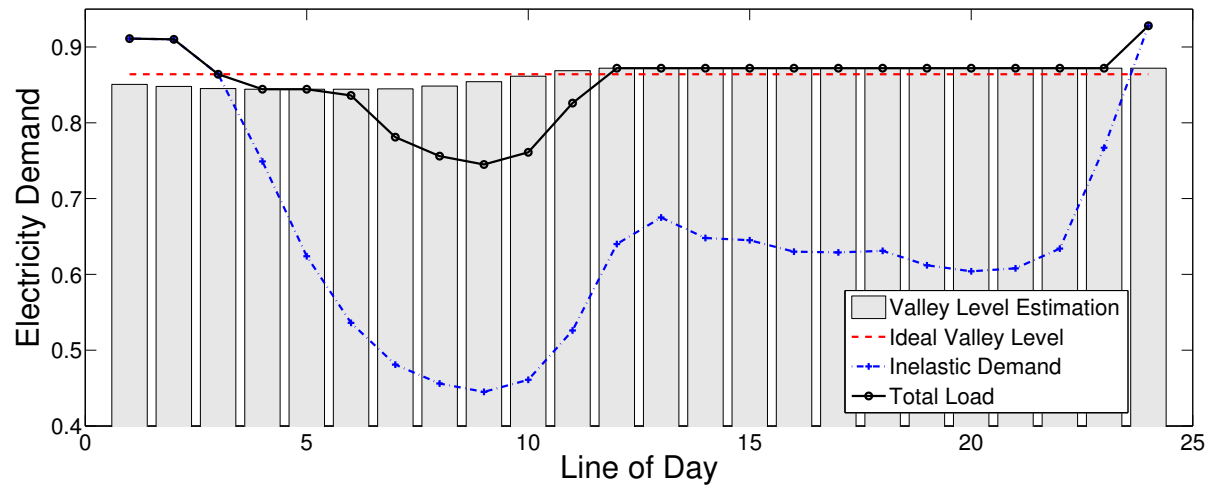

Fig. 2: Illustration of dynamic estimation of valley level [19].

current valley level estimation $\mathbf{a}^{\prime}[t]$, then the next estimation $\mathbf{a}^{\prime}[t+1]$ will be adjusted in the opposite direction to ensure that the "total area" under the curve stays the same. This is illustrated in Fig. 2.

As shown in Fig. 2, the valley level estimation (grey bar) decreases when the total load exceeds the current estimation, increases when the total load is below the estimation, and stays the same when the total load meets the estimation. This behavior is dictated by line 11 , which spread out the current mismatch evenly to the subsequent timeslots. The result below shows that with this dynamic adjustment, the exceptions will not be raised in most cases when the first estimation of the valley level is sufficiently good.

Theorem 4. Assuming that the estimation of $\mathrm{p}_{\mathbf{I}}$ is accurate, and $\mathbf{a}^{\prime}[T-1]-\tilde{\mathbf{p}}[T-1] \in[\underline{\mathbf{r}}[T-1], \overline{\mathbf{r}}[T-1]]$, then the charging profile $(\hat{\mathbf{p}}[1], \ldots, \hat{\mathbf{p}}[T-1])$ obtained from Algorithm 3 without line 6 to 7 is a feasible solution to (5).

With a high EV penetration level, the aggregate charging rate window $[\underline{\mathbf{r}}[T-1], \overline{\mathbf{r}}[T-1]]$ will be large and the condition for Theorem 4 is more likely to hold, and the dynamic adjustment of the valley level $\boldsymbol{\alpha}^{\prime}[t]$ alone is enough to produce a feasible result. Hence, we expect that higher EV penetration will lead to a better performance of Algorithm 3. This effect is shown via simulation in section V-B.

The private information about the arrival and departure of EVs is captured in $\overline{\mathbf{r}}[t]$ and $\underline{\mathbf{r}}[t]$, which is only used locally at charging points for computations in line 6 and line 7 . This private information is not sent to the utility, hence the algorithm is non-intrusive to the privacy of the EV owners. By Theorem 4, when the EV load penetration is large, line 6 and 7 will not be invoked, which means that utility will not be able to infer future charging schedule of EV owners.

As the online adjustment of the valley level (line 11) of Algorithm 3 is trying to make $\hat{\mathbf{p}}[1]+\tilde{\mathbf{p}}[1]+\ldots+\hat{\mathbf{p}}[T-1]+$ $\tilde{\mathbf{p}}[T-1]=(T-1) \mathbf{a}^{\prime}[1]$, the accuracy in estimating $\mathbf{a}^{\prime}[1]$ has a direct impact on the performance of Algorithm 3. The impact of the accuracy of prediction on the performance of Algorithm 3 is shown in section V-D.

\section{Numerical RESUlts}

\section{A. Simulation Setup}

Consider the IEEE 14-bus system shown in Fig. 3 where the circuit specifications and the physical limits are given in the library of the toolbox MATPOWER [27]. The system has five generators connected to buses 1, 2, 3, 6, and 8. Assume that each of the nongenerator bus $4,5,7,9,10,11,12,13$, and 14 is connected to an EV load. Enumerate the batteries of these vehicles as $1,2, \ldots, 9$. Consider that all the batteries are plugged in at time $t=1$ and must be fully charged by time $t=25$, the charging rate of each battery can be controlled only at the discrete time instants $1,2, \ldots, 24$.

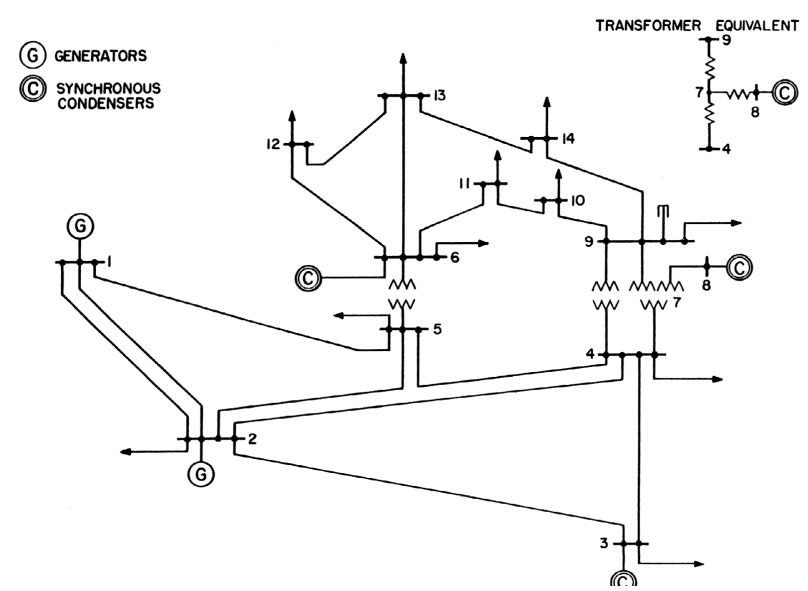

Fig. 3: IEEE 14-bus system studied in Section $\mathrm{V}$ taken from the IEEE power system test archive [28].

Aside from the elastic EV loads, suppose that each bus $k \in$ $\{1,2, \ldots, 14\}$ is also connected to a price-inelastic load given by

$$
(\tilde{\mathbf{p}}[t])_{k}=\frac{l(t) \times P_{k}}{\overline{l(t)}}, \quad t=1,2, \ldots, 24,
$$

where $\left(P_{1}, \ldots, P_{14}\right)$ is equal to the load specificatioin for the IEEE 14-bus system [28], $l(t)$ follows the average residential load in the service area of South California Edison (SCE), a Californian utility, at different times of the day, (cf. SCE website [29]), and $\overline{l(t)}=\sum_{t=1}^{24} l(t)$. The goal is to optimize the controllable parameters of the power network such as the active power supplied by a generator or the charging rate of a battery, which can be modified only at the time instants $1,2, \ldots, 24$. To this end, we aim to minimize the following cost function 


$$
\sum_{t=1}^{24} \sum_{k \in \mathcal{G}} f_{k}\left((\mathbf{p}[t])_{k}\right)+\sum_{t=1}^{24} \sum_{k \in \mathcal{N}} \alpha(\hat{\mathbf{p}}[t])_{k}
$$

This cost function has the following features:

- The power generation cost $f_{k}$ for generation bus $k$ is the quadratic cost specified by IEEE 14 bus test case [28].

- The EV charging cost is independent of its location and invariant over time, and we let $\alpha=2$ in the following.

To compare Algorithm 3 with the optimal solution of (11) solved by the SDP relaxation approach in [9], we used CVX [30], which is a computational package for solving SDP. Three scenarios will be considered in the sequel, and a working example will be shown in Section V-E.

\section{B. Effect of EV penetration}

In this example, we study the effect of EV penetration by varying the proportion of the EV load to the priceinelastic load, and compute the percentage difference. The percentage difference is given by $\left(p_{\text {online }}^{*}-p_{\text {offline }}^{*}\right) / p_{\text {offline }}^{*} \times 100$, where $p_{\text {offline }}^{*}$ and $p_{\text {online }}^{*}$ are the optimal values obtained by Algorithm 2 and Algorithm 3 respectively. Fig. 4 shows the simulation results using three different 24-hour load profiles taken randomly from the SCE residential load data [29]. From Fig. 4, we can make the following observations: firstly,

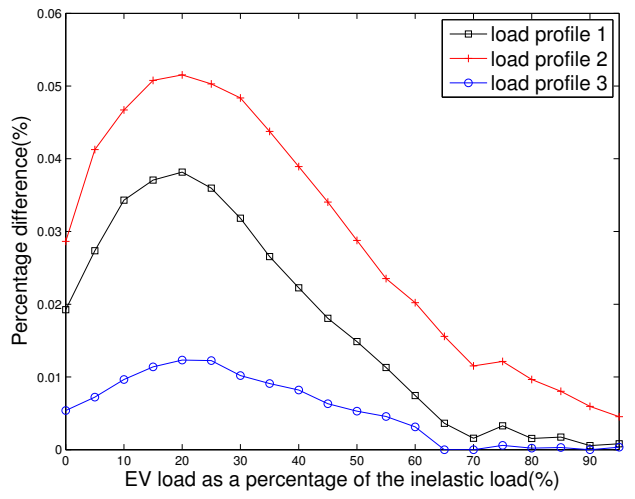

Fig. 4: Profile 1: residential load from 10:00 on Jul. 6th to 9:00 on Jul. 7th; Profile 2: residential load from 15:00 on Aug. 27th to 14:00 on Aug. 28th; Profile 3: residential load from 1:00 on Mar. 11th to 0:00 on Mar. 12th, 2011 [29].

Algorithm 3 solves the joint OPF-EV charging problem (3) almost optimally. From the three randomly chosen load profile, the worst performance is less than $0.06 \%$ different from $p_{\text {offline }}^{*}$. Secondly, we can see all three plots go up initially and then eventually decrease. This is because at the beginning, the EV load is relatively insignificant, Algorithm 2 and 3 perform almost the same as there is little to optimize. As the EV load becomes more significant, the performance gap grows because Algorithm 3 lacks perfect knowledge. However, a higher EV penetration will also lead to a larger room for optimization, as stated in Theorem 4. Hence, the performance gap decreases and eventually approaches zero as the EV penetration increases.

\section{Effect of Controllable Charging Window Size}

In this example, we set the charging rate constraint $\underline{\mathrm{r}}[t]=$ $0, \forall t$, hence, the charging window size corresponds to the upper charging rate limit. Note that larger controllable charging window size corresponds to using smart charging outlet with higher charging rate limit. We plot the graph for the cases when $\overline{\mathbf{r}}[t]$ equal to $5 \%, 10 \%$, and $20 \%$ of the actual EV load respectively. Here, only the residential load profile from 15:00 on Aug. 27th to 14:00 on Aug. 28th is used.

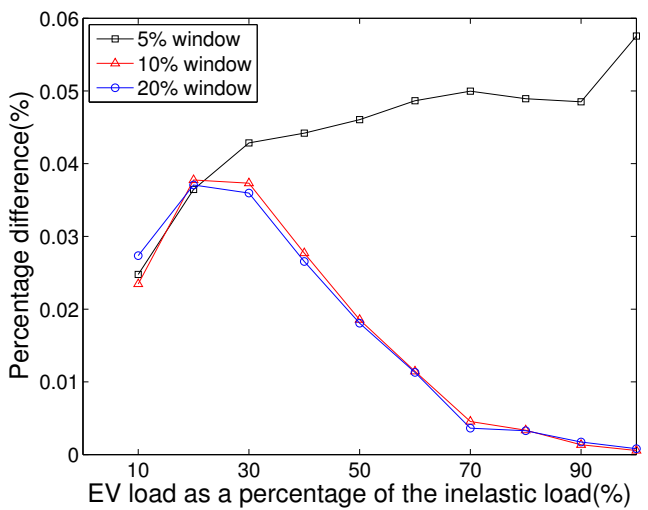

Fig. 5: Effect of varying the charging window size on the performance of Algorithm 3.

From Fig. 5, we can see that a larger allowable charging rate window leads to better results, as the curves corresponding to $10 \%$ and $20 \%$ charging rate window are below that of $5 \%$ charging rate window. However, once the charging rate window is larger than $10 \%$, any additional increase in charging rate window size does not significantly improve the performance.

\section{Effect of Average Load Prediction Accuracy}

In this example, we demonstrate the robustness of Algorithm 3 against prediction error in the prediction of initial valley-level. In Fig. 6, we let the initial estimation of the total average load to be $\mathbf{a}^{\prime}[1]=\left(\tilde{\mathbf{p}_{\mathbf{E}}}+\tilde{\mathbf{p}_{\mathbf{I}}}\right) \times(1+5 i \%), i=$ $1, \ldots, 10$, which corresponds to $5 \%, 10 \%, \ldots, 50 \%$ error in estimation of the total average electricity load. We set the charging rate window to be $20 \%$ of the total EV load. Here, only the residential load profile from 15:00 on Aug. 27th to 14:00 on Aug. 28th is used.

We can see from Fig. 6 that, even when there is $50 \%$ of prediction error in average total electricity demand, the percentage difference to the offline optimal is still less than $1.2 \%$. This shows that Algorithm 3 is robust against prediction error in initial valley level as the suboptimality increase slowly with respect to prediction error for a significant range. Further stress test shows that, only in extreme cases, i.e., when the EV penetration level is greater $80 \%$ and initial estimation error is greater than 90\%, Algorithm 3 will schedule excessive EV load to be charged at the initial first few time slots that leads to the OPF in (4), i.e., finding $\mathbf{W}[t]$, becoming infeasible.

\section{E. A Working Example}

In this example, we use real world residential load variation given in Fig. 7 together with (10) to simulate behavior of 

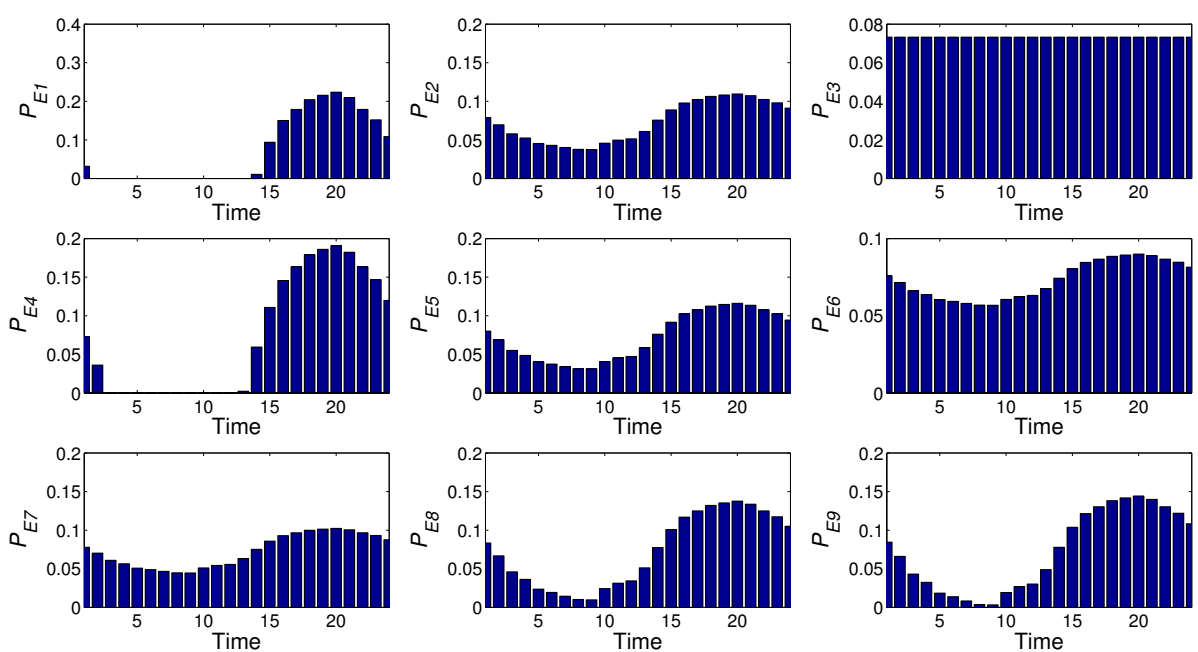

Fig. 8: Optimal EV charging rates for the offline case, $p_{\text {offline }}^{*}=1028$ p.u.
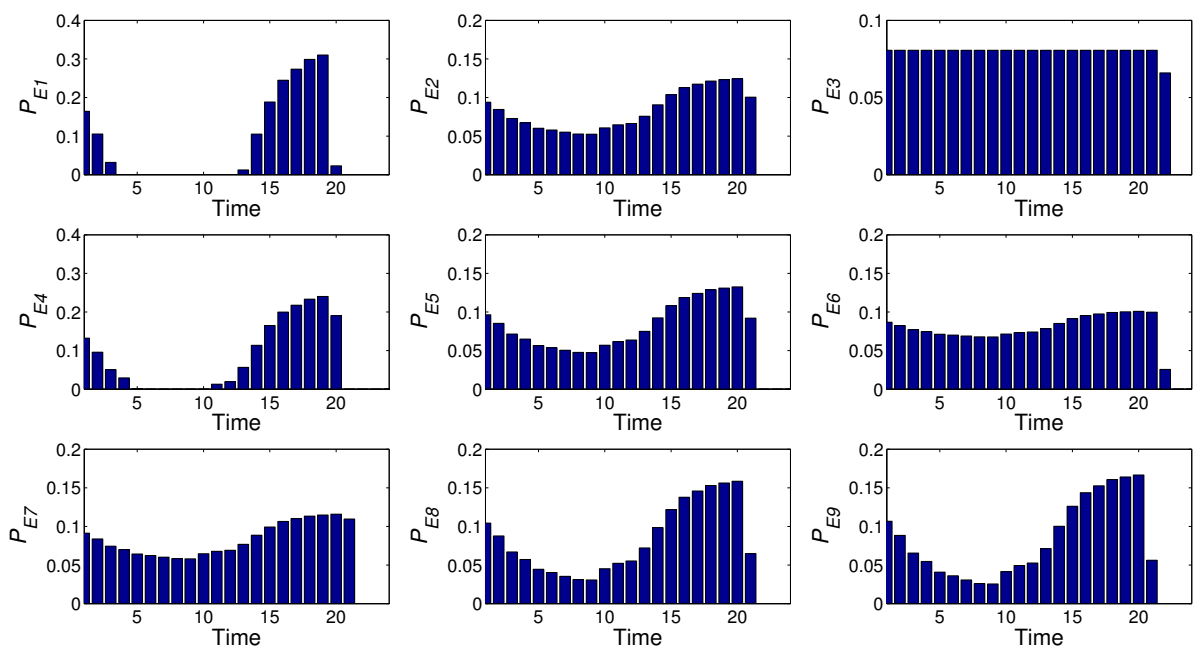

Fig. 9: Optimal EV charging rates for the online case, $p_{\text {online }}^{*}=1031$ p.u.

price-inelastic load. The EV penetration level is set to $50 \%$, and the charging rate window is $20 \%$ of the EV load, and we assume that there is $10 \%$ error in overestimating the initial valley level in the online case. The EV charging profiles produced by Algorithm 2 and Algorithm 3 under this setting are shown in Fig. 8 and Fig. 9 respectively. We can make several observations from Fig. 8 and Fig. 9:

1) The charging rate of each EV is high when the inelastic demand (shown in Fig. 7) is low, which corresponds to the valley-filling characterization.

2) An over-estimation of the initial valley level causes Algorithm 3 to charge the EV batteries faster than optimum. As a result, all the EV batteries are fully charged two time slots before the deadline.

3) The result of Algorithm 3 is still very close to the optimal value, as the percentage difference is small: $\left(p_{\text {online }}^{*}-p_{\text {offline }}^{*}\right) / p_{\text {offline }}^{*} \times 100 \%=0.24 \%$. Hence, in terms of minimizing the quadratic power generation cost, Algorithm 3 performs nearly optimally at this setting.

\section{F. Runtime comparisons}

In this section, we compare the computational time for the SDP optimization approach that uses interior point algorithm to solve the Joint OPF-EV charging problem in [9] with that of Algorithms 2 and 3. The simulation is run on the IEEE 14 bus system, for different scheduling period $T .^{2}$ The computational time measured is the average of running the respective algorithm for ten times.

From Fig. 10, we can see that the time complexity of Algorithm 2 and Algorithm 3 are comparable. Also, both Algorithm 2 and Algorithm 3 have lower time complexity as compared to the SDP optimization method in [9], and the

${ }^{2}$ We used MATLAB version 8.1.0.604 (R2013a), and ran the programs on an Intel Core i7 CPU $2.90 \mathrm{GHz}$ machine. 


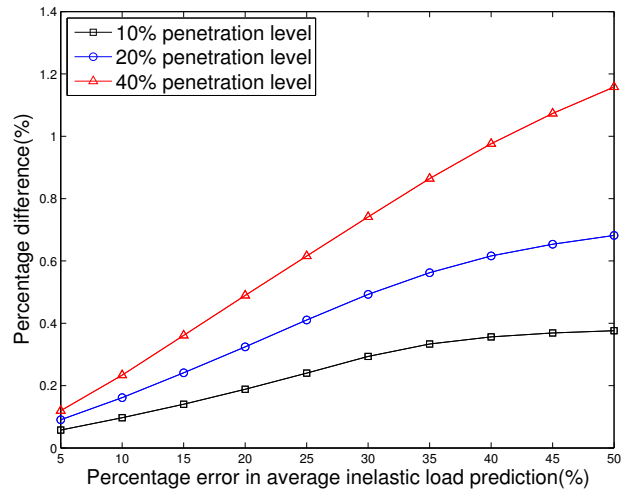

Fig. 6: Effect of percentage error in total average load prediction.

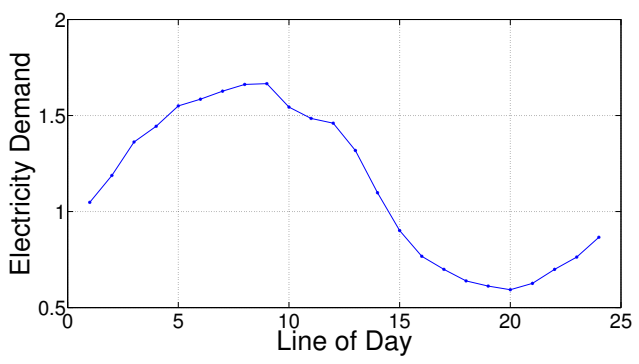

Fig. 7: Variation of price-inelastic load in a 24 hour time frame starting from 10:00 on Jul. 6th 2011 (taken from the SCE website [29]).

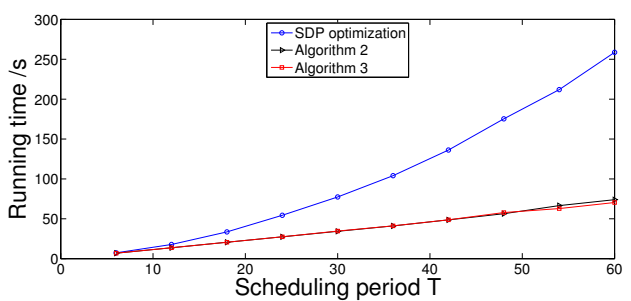

Fig. 10: Runtime comparison between different algorithms

saving in computational time from using Algorithm 2 or Algorithm 3 is more significant as $T$ increases. This demonstrates the advantage of using the valley-filling characterization to decompose the joint OPF-EV charging problem.

\section{CONCLUSION}

We studied a time-dependent OPF charging problem that optimized jointly the operation of the power grid and the charging activity of Electric Vehicles. We proved that this problem is convex with respect to the total electricity demand, characterized the valley-filling charging profile to be optimal under constant electricity price, and proposed a decentralized online algorithm that followed this characterization. At each iteration of the online algorithm, each EV calculated its own charging rate according to the valley level broadcast by the utility, and the utility guided their charging rate by updating the valley level. The online algorithm can be decentralized and thus requires low communication and computation capability. Simulation results showed that the online algorithm performed almost optimally in minimizing power loss, and the optimal value of the online algorithm approached to that of the offline solution as the penetration of EVs increased. However, a higher EV penetration will also lead to a higher sensitivity on the accuracy of estimating the average price-inelastic load.

In this paper, the online algorithm considers a time invariant pricing scheme. However, when there are renewable sources, electricity prices can vary in real time. In addition, EVs arrivals and departures may be stochastic. Incorporating real time pricing, modeling EV arrivals as random events and accounting for the uncertainties are interesting directions for future research. Furthermore, our results can be generalized to more complex joint OPF-EV charging problems, e.g., ramp rate constraints on the power generators. Extending the algorithm to consider reactive power (e.g., using rectifiers) may also be an interesting area for future research.

\section{APPENDIX}

\section{A. Analysis of Valley-filling Characterization}

In this section, we prove Theorem 1, Lemma 1 and Theorem 2 respectively.

Proof of Theorem 1: Let $\hat{\mathbf{p}}[\mathbf{t}]+\tilde{\mathbf{p}}[\mathbf{t}]$ be the argument to $F(\cdot)$ in (4). From [5], with strong duality, $F(\hat{\mathbf{p}}[t]+\tilde{\mathbf{p}}[t])$ is equal to the optimal value of the following problem:

$$
\begin{array}{ll}
\text { maximize } & h(\boldsymbol{\Theta}, \hat{\mathbf{p}}[t]+\tilde{\mathbf{p}}[t]) \\
\text { subject to } & A(\boldsymbol{\Theta}) \succeq 0,
\end{array}
$$

where $\Theta$ is the set of dual variables corresponding to the different physical constraints in (3) without the rank constraint (3h). $h(\boldsymbol{\Theta}, \hat{\mathbf{p}}[t]+\tilde{\mathbf{p}}[t])$ is the Lagrange dual function (in fact, an affine function in $\hat{\mathbf{p}}[t]+\tilde{\mathbf{p}}[t]$ ) for every feasible $\boldsymbol{\Theta}$, and $A(\boldsymbol{\Theta})$ is a linear matrix inequality constraint in $\Theta$ (cf. the definition of Optimization 4 in [5]). Let $\mathbb{D}$ denote the feasible set of $\Theta$ that satisfies the constraint (12b). Then,

$$
F(\hat{\mathbf{p}}[t]+\tilde{\mathbf{p}}[t])=\sup _{\boldsymbol{\Theta} \in \mathbb{D}}(h(\boldsymbol{\Theta}, \hat{\mathbf{p}}[t]+\tilde{\mathbf{p}}[t])),
$$

which is a convex function in $(\hat{\mathbf{p}}[t]+\tilde{\mathbf{p}}[t])$ as it is a pointwise supremum of convex functions [31].

Proof of Lemma 1: $\sum_{t=1}^{T-1} \boldsymbol{\alpha}^{\boldsymbol{\top}} \hat{\mathbf{p}}[t]=\boldsymbol{\alpha}^{\boldsymbol{\top}} \mathbf{c}$, which is a constant. Also, by Jensen's inequality, we have

$$
\begin{aligned}
& \frac{F(\hat{\mathbf{p}}[1]+\tilde{\mathbf{p}}[1]) \ldots+F(\hat{\mathbf{p}}[T-1]+\tilde{\mathbf{p}}[T-1])}{T-1} \\
\geq & F\left(\frac{\hat{\mathbf{p}}[1]+\tilde{\mathbf{p}}[1]+\ldots+\hat{\mathbf{p}}[T-1]+\tilde{\mathbf{p}}[T-1]}{T-1}\right) \\
& =F\left(\frac{\mathbf{c}+\mathbf{d}}{T-1}\right),
\end{aligned}
$$

where the equality holds when $\hat{\mathbf{p}}[1]+\tilde{\mathbf{p}}[1]=\ldots=\hat{\mathbf{p}}[T-1]+$ $\tilde{\mathbf{p}}[T-1]=(\mathbf{c}+\mathbf{d}) /(T-1)$.

To prove Theorem 2, we start with the following:

Definition 2. Denote $a>(b, c)>d$ to mean both $a>$ $\max (b, c)$ and $d<\min (b, c)$.

Lemma 2. Let $f$ to be a convex function, and $a+d=b+c$ and $a>(b, c)>d$, then $f(a)+f(d) \geq f(b)+f(c)$.

Proof: Without loss of generality, assume that $a>b>$ $c>d$, as $a+d=b+c$, then there exists a $k \in(0,1)$ such 
that $k a+(1-k) c=b$, and $k d+(1-k) b=c$. By convexity of $f$, we have

$$
\begin{aligned}
& k f(a)+(1-k) f(c) \geq f(b) \\
& k f(d)+(1-k) f(b) \geq f(c) .
\end{aligned}
$$

Summing (15a) and (15b) completes the proof.

Proof of Theorem 2: Consider all the arguments to $F(\cdot)$ to be scalars first, we can get the proof for the vector case by repeating the argument for each component of the vector. Suppose $\left(\hat{p}^{\prime}[1], \ldots, \hat{p}^{\prime}[T-1]\right)$ is an optimal solution to the scalar case of $(5)$, and let $(\hat{p}[1], \ldots, \hat{p}[T-1])$ be the valleyfilling load.

Suppose we have some $\hat{p}^{\prime}[j] \neq \hat{p}[j]$, without loss of generality, assume $\hat{p}^{\prime}[j]>\hat{p}[j]$, by (5c), there must exist some $k$, such that $\hat{p}^{\prime}[k]<\hat{p}[k]$. From Definition 1, a valley-filling profile has the minimal deviation from the flat profile, i.e., for $(5)$, if $(\hat{p}[1], \ldots, \hat{p}[T-1])$ is a valley-filling profile, and $\left(\hat{p}^{\prime}[1], \ldots, \hat{p}^{\prime}[T-1]\right)$ is any feasible charging profile, then $|a-((\hat{p}[i])+(\tilde{p}[i]))| \leq\left|a-\left(\left(\hat{p}^{\prime}[i]\right)+\left(\tilde{p}^{\prime}[i]\right)\right)\right|, \quad \forall i$. Hence,

$$
\begin{aligned}
\hat{p}[j]<\hat{p}^{\prime}[j] & \Rightarrow \hat{p}[j]+\tilde{p}[j]<\hat{p}^{\prime}[j]+\tilde{p}[j] \\
& \Rightarrow \hat{p}^{\prime}[j]+\tilde{p}[j]>a, \\
\hat{p}[k]<\hat{p}^{\prime}[k] & \Rightarrow \hat{p}[k]+\tilde{p}[k]>\hat{p}^{\prime}[k]+\tilde{p}[k] \\
& \Rightarrow \hat{p}^{\prime}[k]+\tilde{p}[k]<a .
\end{aligned}
$$

We consider two cases, $\hat{p}^{\prime}[j]-\hat{p}[j] \leq \hat{p}[k]-\hat{p}^{\prime}[k]$ and $\hat{p}^{\prime}[j]-$ $\hat{p}[j]>\hat{p}[k]-\hat{p}^{\prime}[k]$ separately.

Case 1 , suppose that $\hat{p}^{\prime}[j]-\hat{p}[j] \leq \hat{p}[k]-\hat{p}^{\prime}[k]$, meaning that $\hat{p}^{\prime}[j]$ deviates from the valley-filling load by a smaller amount. In this case, we swap $\hat{p}^{\prime}[j]$ and $\hat{p}^{\prime}[k]$ by $\hat{p}^{\prime \prime}[j]$ and $\hat{p}^{\prime \prime}[k]$ defined as follows:

$$
\hat{p}^{\prime \prime}[j]=\hat{p}[j], \quad \hat{p}^{\prime \prime}[k]=\hat{p}^{\prime}[k]+\hat{p}^{\prime}[j]-\hat{p}[j] .
$$

Now, $\hat{p}^{\prime \prime}[k]$ is feasible because $\hat{p}^{\prime}[k] \leq \hat{p}^{\prime \prime}[k] \leq \hat{p}[k]$ by assumption. Furthermore, $\hat{p}^{\prime \prime}[j]+\hat{p}^{\prime \prime}[k]=\hat{p}^{\prime}[j]+\hat{p}^{\prime}[k]$. From (16) and (17), we have

$$
\hat{p}^{\prime}[k]+\tilde{p}[k] \leq\left(\hat{p}^{\prime \prime}[k]+\tilde{p}[k], \hat{p}^{\prime \prime}[j]+\tilde{p}[j]\right) \leq \hat{p}^{\prime}[j]+\tilde{p}[j] .
$$

Hence, by Lemma 2, we have

$$
\begin{aligned}
& F\left(\hat{p}[j]^{\prime \prime}+\tilde{p}[j]\right)+F\left(\hat{p}[k]^{\prime \prime}+\tilde{p}[k]\right) \\
\leq & F\left(\hat{p}^{\prime}[j]+\tilde{p}[j]\right)+F\left(\hat{p}^{\prime}[k]+\tilde{p}[k]\right),
\end{aligned}
$$

which means that applying such swapping procedure will not increase the objective value of (5).

Case 2, suppose that $\hat{p}^{\prime}[j]-\hat{p}[j]>\hat{p}[k]-\hat{p}^{\prime}[k]$. Then in this case apply the swapping procedure as follows:

$$
\hat{p}^{\prime \prime}[j]=\hat{p}^{\prime}[j]+\hat{p}^{\prime}[k]-\hat{p}[k], \quad \hat{p}^{\prime \prime}[k]=\hat{p}^{\prime}[k] .
$$

By a symmetric argument to case 1, applying such a swapping procedure will not decrease the objective value of (5) as well.

Note that each time a swapping is applied, there will be at least one fewer $\hat{p}^{\prime}[j]$ that deviates from the valley-filling load. Applying a finite number of this swapping procedure completes the proof.

\section{B. Analysis of Algorithm 3}

In this section, we prove Theorem 3 and Theorem 4.

Proof of Theorem 3: Firstly, the charging profile $(\hat{\mathbf{p}}[1], \ldots, \hat{\mathbf{p}}[T-1])$ will not overcharge EV batteries, i.e., $\sum_{k=1}^{t} \hat{\mathbf{p}}[k] \leq \mathbf{c} \forall t$, by line 7 to 8 . Secondly, it will not undercharge, i.e., $\sum_{t=1}^{T-1} \hat{\mathbf{p}}[t] \geq \mathbf{c}$ because of line 5 to 6 . Hence, $\sum_{t=1}^{T-1} \hat{\mathbf{p}}[t]=\mathbf{c}$, the charging sum constraint is satisfied. Thirdly, assuming the charging rate constraints permit a feasible solution, then $\underline{\mathbf{r}}[t] \leq \hat{\mathbf{p}}[t] \leq \overline{\mathbf{r}}[t], \forall t$. This can be proved by contradiction, suppose the charging profile $(\hat{\mathbf{p}}[1], \ldots, \hat{\mathbf{p}}[T-1])$ obtained from the above algorithm has some $i, j$, such that $(\hat{\mathbf{p}}[t])_{j}<(\underline{\mathbf{r}}[t])_{j}$, note that $(\hat{\mathbf{p}}[t])_{j}$ can only go below $(\underline{\mathbf{r}}[t])_{j}$ if the condition in line 7 is true. Pick the smallest $t$ where the charging rate constraint is violated. If $t=1$, then that means $\sum_{t=1}^{T-1}(\underline{\mathbf{r}}[t])_{j}>\mathbf{c}_{j}$ by line 7 , i.e., the charging limit does not permit a feasible solution, which is a contradiction. If $t>1$, then by line 8 , we have $(\hat{\mathbf{p}}[t])_{j}=\mathbf{c}_{j}-\sum_{l=t+1}^{T-1}(\underline{\mathbf{r}}[l])_{j}-\sum_{k=1}^{t-1}(\hat{\mathbf{p}}[k])_{j}<(\underline{\mathbf{r}}[t])_{j}$, and after rearranging, we have $\sum_{k=1}^{t-1}(\hat{\mathbf{p}}[k])_{j}>\mathbf{c}_{j}-\sum_{l=t}^{T-1}(\underline{\mathbf{r}}[l])_{j}$, but that is not possible, because at the end of iteration $t-1$, line 7 to line 8 will always make sure that $\sum_{k=1}^{t-1}(\hat{\mathbf{p}}[k])_{j} \leq$ $\mathbf{c}_{j}-\sum_{l=t}^{T-1}(\underline{\mathbf{r}}[l])_{j}$. The other case where the upper charging rate constraint is violated can be proved in a similar way. Therefore, when both the charging sum and the charging rate constraints are satisfied, the charging profile produced by Algorithm 3 is a feasible solution to (5).

Proof of Theorem 4: Firstly, $\mathbf{a}^{\prime}[T-1]-\tilde{\mathbf{p}}[T-1] \in[\underline{\mathbf{r}}[T-$ $1], \overline{\mathbf{r}}[T-1]] \Rightarrow \hat{\mathbf{p}}[T-1]+\tilde{\mathbf{p}}[T-1]=\mathbf{a}^{\prime}[T-1]$. To prove that $(\hat{\mathbf{p}}[1], \ldots, \hat{\mathbf{p}}[T-1])$ is a feasible solution to (5), we only need to show that $\sum_{t=1}^{T-1}(\hat{\mathbf{p}}[t]+\tilde{\mathbf{p}}[t])=(T-1)\left(\mathbf{p}_{\mathbf{E}}+\mathbf{p}_{\mathbf{I}}\right)=$ $(T-1) \mathbf{a}^{\prime}[1]$. Note that $\hat{\mathbf{p}}[T-1]+\tilde{\mathbf{p}}[T-1]=\mathbf{a}^{\prime}[T-1]$. If $T=2$ then we are done, otherwise repeatedly applying line 11 of Algorithm 3, we will have

$$
\hat{\mathbf{p}}[1]+\tilde{\mathbf{p}}[1]+\ldots+\hat{\mathbf{p}}[T-1]+\tilde{\mathbf{p}}[T-1]=(T-1) \mathbf{a}^{\prime}[1] .
$$

Hence $\sum_{t=1}^{T-1}(\hat{\mathbf{p}}[t]+\tilde{\mathbf{p}}[t])=(T-1) \mathbf{a}^{\prime}[1]$, which is what we set out to prove.

\section{ACKNOWLEDGEMENT}

The authors gratefully acknowledge helpful discussions with Steven H. Low at Caltech.

\section{REFERENCES}

[1] K. Clement-Nyns, E. Haesen, and J. Driesen, "The impact of charging plug-in hybrid electric vehicles on a residential distribution grid," IEEE Trans. Power Syst., vol. 25, no. 1, pp. 371-380, February 2010.

[2] S. Han, S. Han, and K. Sezaki, "Development of an optimal vehicleto-grid aggregator for frequency regulation," IEEE Trans. Smart Grid, vol. 1 , no. 1 , pp. 65-72, June 2010.

[3] A. Boulanger, A. Chu, S. Maxx, and D. Waltz, "Vehicle electrification: Status and issues," Proc. IEEE, vol. 99, no. 6, pp. 1116-1138, June 2011.

[4] O. Sundstrom and C. Binding, "Flexible charging optimization for electric vehicles considering distribution grid constraints," IEEE Trans. Smart Grid, vol. 3, no. 1, pp. 26 -37, March 2012.

[5] J. Lavaei and S. Low, "Zero duality gap in optimal power flow problem," IEEE Trans. Power Syst., vol. 27, no. 1, pp. 92 -107, February 2012.

[6] S. Bose, D. F. Gayme, S. Low, and K. M. Chandy, "Optimal power flow over tree networks," in Proc. IEEE Allerton Conf. on Communication, Control and Computing, Monticello, September 2011. 
[7] N. Li, L. Chen, and S. Low, "Exact convex relaxation of OPF for radial networks using branch flow model," in Proc. IEEE Conf. on Smart Grid Communications, Taiwan, Nov 2012, pp. 7-12.

[8] C. W. Tan, D. W. H. Cai, and X. Lou, "Resistive network optimal power flow: Uniqueness and algorithms," IEEE Trans. Power Syst., vol. to be published, 2014

[9] S. Sojoudi and S. Low, "Optimal charging of plug-in hybrid electric vehicles in smart grids," in Proc. IEEE Power and Energy Society General Meeting, Detroit, July 2011, pp. 1-6.

[10] X. Lou and C. W. Tan, "Optimization decomposition of resistive power networks with energy storage," IEEE Journal on Selected Areas in Comms., vol. to be published, 2014.

[11] S. Chen and L. Tong, "iEMS for large scale charging of electric vehicles: Architecture and optimal online scheduling," in Proc. IEEE Conf. on Smart Grid Communications. Taiwan: IEEE, 2012, pp. 629-634.

[12] S. Chen, Y. Ji, and L. Tong, "Large scale charging of electric vehicles," in IEEE Power and Energy Society General Meeting, 2012, pp. 1-9.

[13] L. Gan, U. Topcu, and S. Low, "Optimal decentralized protocol for electric vehicle charging," IEEE Trans. Power Syst., vol. 28, no. 2, pp. 940-951, 2013.

[14] L. Gan, A. Wierman, U. Topcu, N. Chen, and S. H. Low, "Real-time deferrable load control: handling the uncertainties of renewable generation," in Proceedings of the Fourth International Conference on Future Energy Systems, ser. e-Energy '13. New York, NY, USA: ACM, 2013, pp. 113-124. [Online]. Available: http://doi.acm.org/10.1145/2487166.2487179

[15] O. Ardakanian, C. Rosenberg, and S. Keshav, "Distributed control of electric vehicle charging," in Proceedings of the Fourth International Conference on Future Energy Systems. ACM, 2013, pp. 101-112.

[16] W. Lee, L. Xiang, R. Schober, and V. W. Wong, "Analysis of the behavior of electric vehicle charging stations with renewable generations," in Proc. IEEE Conf. on Smart Grid Communications. Vancouver: IEEE, 2013, pp. 145-150.

[17] A. Lam, Y.-W. Leung, and X. Chu, "Electric vehicle charging station placement," in Proc. IEEE Conf. on Smart Grid Communications. Vancouver: IEEE, 2013, pp. 510-515.

[18] M. H. Albadi and E. El-Saadany, "Demand response in electricity markets: An overview," in IEEE Power Engineering Society General Meeting, June 2007, pp. 1-5.

[19] N. Chen, T. Quek, and C. W. Tan, "Optimal charging of electric vehicles in smart grid: Characterization and valley-filling algorithms," in Proc. IEEE Conf. on Smart Grid Communications, Tainan, November 2012, pp. $13-18$

[20] L. Chen, N. Li, L. Jiang, and S. H. Low, "Optimal demand response: problem formulation and deterministic case," Control and Optimization Methods for Electric Smart Grids, pp. 63-85, 2012.

[21] F. Blaabjerg, R. Teodorescu, M. Liserre, and A. Timbus, "Overview of control and grid synchronization for distributed power generation systems," Industrial Electronics, IEEE Transactions on, vol. 53, no. 5, pp. 1398-1409, Oct 2006.

[22] A. Ipakchi and F. Albuyeh, "Grid of the future," IEEE Power Energy Mag., vol. 7, no. 2, pp. 52-62, March-April 2009.

[23] A. Bergen and V. Vittal, Power Systems Analysis. Prentice Hall, 2000.

[24] L. Vandenberghe and S. Boyd, "Semidefinite programming," SIAM Review, vol. 38, pp. 49-95, 1996.

[25] L. Porkolab and L. Khachiyan, "On the complexity of semidefinite programs," Journal of Global Optimization, vol. 10, pp. 351-365, Jun 1997.

[26] T. Cover and J. Thomas, Elements of Information Theory. WileyInterscience, 2006.

[27] R. D. Zimmerman, C. E. Murillo-Sánchez, and R. J. Thomas, "MATPOWER: Steady-state operations, planning and analysis tools for power systems research and education," IEEE Trans. Power Syst., vol. 26, no. 1, pp. 12-19, February 2011.

[28] R. Christie, "Power systems test case archive." [Online]. Available: http://www.ee.washington.edu/research/pstca

[29] "SCE website." [Online]. Available: http://www.sce.com/005_regul_ info/eca/DOMSM11.DLP

[30] M. Grant and S. Boyd, "CVX: Matlab software for disciplined convex programming, version 1.21," http://cvxr.com/cvx, April 2011.

[31] S. Boyd and L. Vandenberghe, Convex Optimization. Cambridge University Press, 2004.

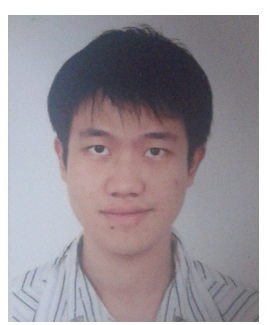

Niangjun Chen (S'13) received the B.A. (Hons.) degree in computer science from University of Cambridge, UK, in 2011. He is currently a Ph.D. student in computer science at California Institute of Technology, Pasadena.

From July 2009 to August 2010, he was a Research Engineer with the Institute for Infocomm Research, Singapore. His current research interests include load control, online algorithms and optimization in smart grids.

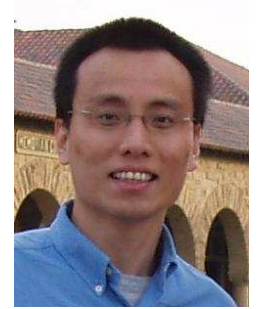

Chee Wei Tan (M'08-SM'12) received the M.A and Ph.D. degrees in electrical engineering from Princeton University, Princeton, NJ, in 2006 and 2008, respectively. He is an Assistant Professor at the City University of Hong Kong. Previously, he was a Postdoctoral Scholar at the California Institute of Technology (Caltech), Pasadena, CA. He was a Visiting Faculty at Qualcomm R\&D, San Diego, CA, in 2011. His research interests are in networks, inference in online large data analytics, and optimization theory and its applications.

Dr. Tan was the recipient of the 2008 Princeton University Wu Prize for Excellence and was awarded the 2011 IEEE Communications Society AP Outstanding Young Researcher Award. He was a selected participant at the U.S. National Academy of Engineering China-America Frontiers of Engineering Symposium in 2013

Dr. Tan currently serves as an Editor for the IEEE TRANSACTIONS ON COMMUNICATIONS and the Chair of the IEEE Information Theory Society Hong Kong Chapter. He was a Symposium Co-Chair for the 2013 IEEE International Conference on Smart Grid Communications.

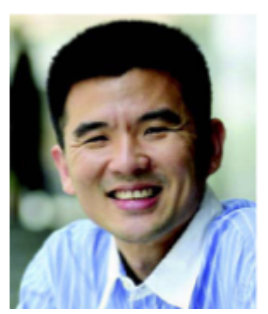

Tony Q.S. Quek (S'98-M'08-SM'12) received the B.E. and M.E. degrees in Electrical and Electronics Engineering from Tokyo Institute of Technology, Tokyo, Japan, respectively. At Massachusetts Institute of Technology, he earned the Ph.D. in Electrical Engineering and Computer Science. Currently, he is an Assistant Professor with the Information Systems Technology and Design Pillar at Singapore University of Technology and Design (SUTD). He is also a Scientist with the Institute for Infocomm Research. His main research interests are the application of mathematical, optimization, and statistical theories to communication, networking, signal processing, and resource allocation problems. Specific current research topics include sensor networks, heterogeneous networks, green communications, smart grid, wireless security, compressed sensing, big data processing, and cognitive radio.

Dr. Quek has been actively involved in organizing and chairing sessions, and has served as a member of the Technical Program Committee as well as symposium chairs in a number of international conferences. He is serving as the TPC co-chair for IEEE ICCS in 2014, the Wireless Networks and Security Track for IEEE VTC Fall in 2014, the PHY \& Fundamentals Track for IEEE WCNC in 2015, and the Communication Theory Symposium for IEEE ICC in 2015. He is currently an Editor for the IEEE TRANSACTIONS ON COMMUNICATIONS, the IEEE WIRELESS COMMUNICATIONS LETTERS, and an Executive Editorial Committee Member for the IEEE TRANSACTIONS ON WiRELESS COMMUNICATIONS. He was Guest Editor for the IEEE Communications Magazine (Special Issue on Heterogeneous and Small Cell Networks) in 2013 and the IEEE SignAL PROCESSING MAGAZINE (Special Issue on Signal Processing for the 5G Revolution) in 2014.

Dr. Quek was honored with the 2008 Philip Yeo Prize for Outstanding Achievement in Research, the IEEE Globecom 2010 Best Paper Award, the 2011 JSPS Invited Fellow for Research in Japan, the CAS Fellowship for Young International Scientists in 2011, the 2012 IEEE William R. Bennett Prize, and the IEEE SPAWC 2013 Best Student Paper Award. 